\title{
A97-37021
}

AIAA-97-3472

\section{ANALYTICAL SOLUTION OF MISSILE TERMINAL GUIDANCE}

\author{
Tetsuya Takehira, Nguyen X. Vinh ${ }^{\dagger}$ and Pierre T. Kabamba ${ }^{\ddagger}$ \\ The University of Michigan \\ Ann Arbor, MI 48109-2118, U.S.A.
}

\begin{abstract}
A new guidance law, which combines pursuit guidance and proportional navigation is proposed. This guidance law depends on two parameters that determine the relative importance of pursuit guidance and proportional navigation. Numerical simulations of the nonlinear equations of motion suggest that the parameters of this law can be chosen to reduce the peak value of the missile acceleration. When the engagement ends in a tail chase, and linearization is valid, the linearized equations of motion lead to a confluent hypergeometric equation. This equation is solved in closed form, in the general case where the target performs maneuvers such that its heading angle is a polynomial function of time. The analytic solution based on linearization and the numerical simulation of the nonlinear equations show good agreement.
\end{abstract}

\section{Nomenclature}

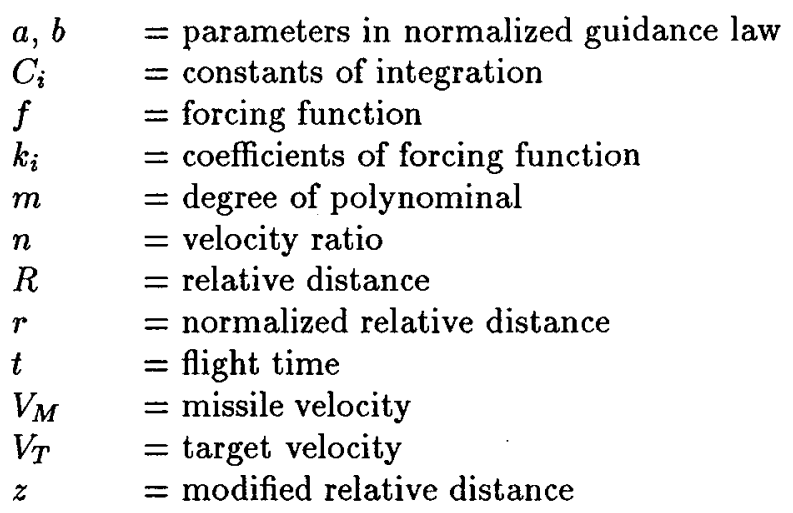

\footnotetext{
*Doctoral Candidate, Department of Aerospace Engineering, Student Member AIAA

${ }^{\dagger}$ Professor, Department of Aerospace Engineering, Member AIA A

¡Professor, Department of Aerospace Engineering

Copyright (C) 1997 by the American Institute of Aeronautics and Astronautics, Inc. All right reserved.
}

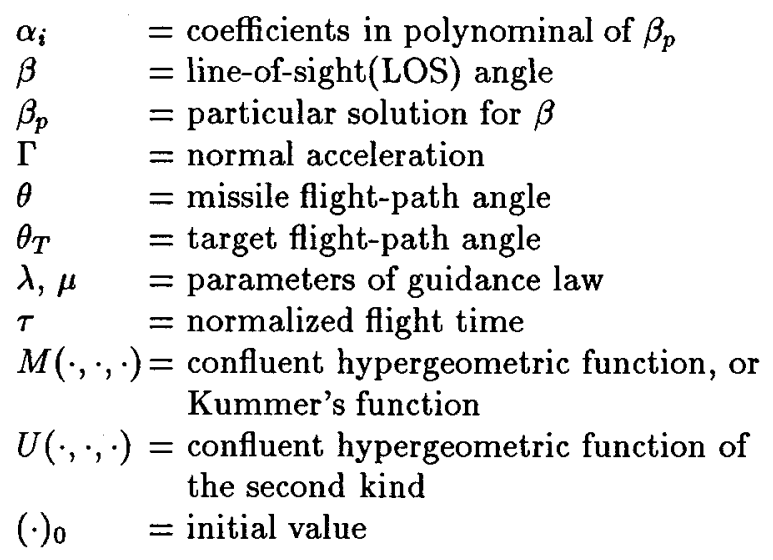

\section{Introduction}

This paper considers the standard problem of guiding a craft or a missile on a collision course to a maneuvering target. Among the known guidance laws, the simplest one is pursuit guidance which constantly directs the velocity of the missile towards the moving target. This law only requires knowledge of the missile-target line-of-sight. Another practical guidance law is proportional navigation, in which the missile detects any rotation of the line-of-sight and applies a turning rate proportional to this rotation. The relative advantages and disadvantages of these guidance laws are well known and presented in many texts ${ }^{[1]-[8]}$. Various aspects of proportional navigation are topics of discussion in the current literature ${ }^{[9]-[16]}$.

Guelman[9] obtained a closed-form solution of true proportional navigation for a nonmaneuvering target. Shukla et al.[10] derived a general form of linearized solution to proportional navigation. Various solutions for pure proportional navigation were given by Mahapatra et al.[11] and Becker[12]. Cochran et al.[13] obtained new closed-form solution in terms of elliptic functions and integrals. Yuan et al.[14]-[15] 
also presented closed-from solutions of true proportional navigation for both maneuvering and nonmaneuvering targets and solution of generalized proportional navigation. Balakrishnan [16] introduced a class of proportional navigation laws through an approximation of time-to-go and a transformation of state variables.

In this paper we propose a guidance law which is a combination of pursuit and proportional navigation, with the aim of retaining the effectiveness of pure pursuit and at the same time reducing the lateral acceleration, which is a characteristic feature of proportional navigation. After formulation of the problem, the effectiveness of the control law is tested numerically for various maneuvers of the target. It is shown that by adjusting two parameters which control the turning rate of the missile, it is possible to reduce the peak acceleration to an acceptable level. Furthermore, in the final and most important phase of the interception, linearization of the dynamics leads to a confluent hypergeometric equation which is solvable analytically when the heading angle of the target is a polynominal function of a dimensionless independent variable.

\section{Equations of Motions}

For the case of planar pursuit-evasion, we have the equations

$$
\begin{aligned}
& \frac{d R}{d t}=V_{T} \cos \left(\beta-\theta_{T}\right)-V_{M} \cos (\beta-\theta) \\
& \frac{d \beta}{d t}=\frac{1}{R}\left[-V_{T} \sin \left(\beta-\theta_{T}\right)+V_{M} \sin (\beta-\theta)\right]
\end{aligned}
$$

where $R$ and $\beta$ are the relative distance between target and missile positions and line-of-sight angle, and $\theta$ and $\theta_{T}$ are missile and target flight path angle, respectively. We use the flight time $t$ as the independent variable. The geometry of the interception is shown in Fig.1.

To control the missile towards the intercept point, we use the following new guidance law

$$
\frac{d \theta}{d t}=\lambda \frac{d \beta}{d t}+\mu \sin (\beta-\theta),
$$

where $\lambda$ and $\mu$ are constant parameters. The first term on the right hand side in this guidance equation represents proportional navigation, and the second term represents pursuit guidance. Therefore this guidance law is constructed by combining the two fundamental guidance laws through the use of the two parameters $\lambda$ and $\mu$.

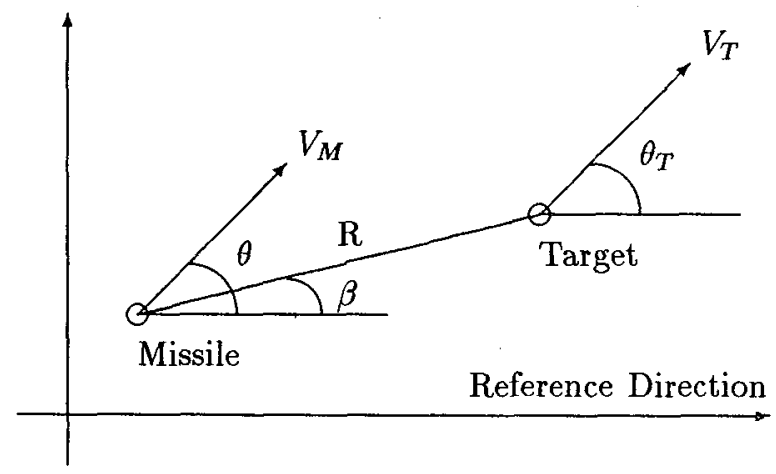

Figure 1: Geometry of Interception.

To normalize the equations, the following nondimensional variables are defined for the case of constant speed of the target. We have

$$
\begin{aligned}
\tau & =\frac{V_{T}}{R_{0}} t, \\
r & =\frac{R}{R_{0}} \\
n & =\frac{V_{M}}{V_{T}} \\
a & =\lambda, \\
b & =\frac{R_{0}}{V_{T}} \mu,
\end{aligned}
$$

where $R_{0}$ is the initial relative distance, $\tau$ is the normalized time, $r$ is the normalized relative distance, $a$ and $b$ are parameters of the normalized guidance law and $n$ is the velocity ratio.

Using these non-dimensional variables, the normalized equations are

$$
\begin{aligned}
& \frac{d r}{d \tau}=\cos \left(\beta-\theta_{T}\right)-n \cos (\beta-\theta), \\
& \frac{d \beta}{d \tau}=\frac{1}{r}\left[-\sin \left(\beta-\theta_{T}\right)+n \sin (\beta-\theta)\right], \\
& \frac{d \theta}{d \tau}=a \frac{d \beta}{d \tau}+b \sin (\beta-\theta) .
\end{aligned}
$$

\section{Effectiveness of Guidance Law}

Although we have restricted the analysis to the case of constant speed for the target, the nonlinear equations (5) (7) allow its maneuverability through the 
arbitrary change in the heading $\theta_{T}(\tau)$ as a function of the dimensionless time, and the possibility of modulating the speed ratio $n(\tau)$ for the missile. Of course, the turning rate of the missile is dictated by the guidance law (7), now written in dimensionless form with two arbitrary constants $a$ and $b$. For this paper, we use a constant value for $n$.

To show the effectiveness of the guidance law, and the influence of the parameters $a$ and $b$ on its performance, we consider the complete non-linear system and generate the intercept trajectory numerically.

Without loss of generality, the reference direction is taken along the initial target velocity. Hence, we have at the initial time

$$
\theta_{T}(0)=0, \quad \beta(0)=\beta_{0}, \quad r(0)=1, \quad \theta(0)=\theta_{0} .
$$

In the first two examples, we use a constant speed ratio $n=3.0$, and $\beta_{0}=1 / 20$, while varying $\theta_{0}$ from 0 to $\pi / 2$. In the last example, we use $n=1.701$

a / In the simplest case, we keep constant the heading of the target. Hence

$$
\theta_{T}=0 .
$$

Figure 2 shows the intercept trajectories for various initial headings of the missile, using $\theta_{0}=$ $0, \pi / 6, \pi / 3, \pi / 2$.

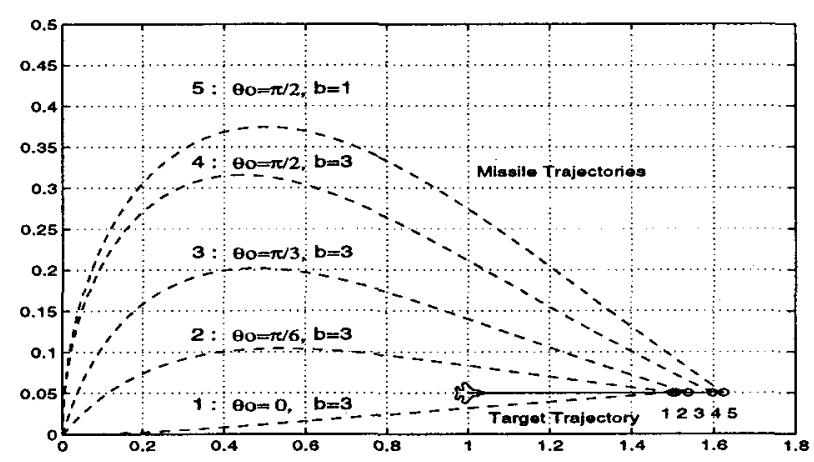

Figure 2: Intercept Trajectories for NonManeuvering Target.

As expected, for the same set of values $a=3.0$, $b=3.0$, the tail chase case $\theta_{0}=0$, is the fastest, and as $\theta_{0}$ increases, it takes longer time for the interception. The interception points are denoted in order of increasing time and range in the figure. The normal acceleration $\Gamma$, in normalized form, is

$\Gamma=n \frac{d \theta}{d \tau}=-\frac{a n}{r} \sin \left(\beta-\theta_{T}\right)+n\left(\frac{a n}{r}+b\right) \sin (\beta-\theta)$.

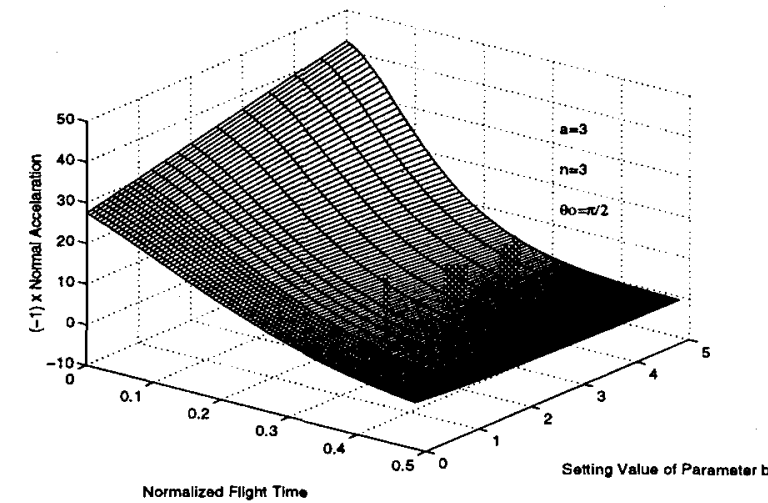

Figure 3: Time History of Normal Accelerations $\Gamma$ vs. Setting Values of Parameter $b$.

It is highest at the initial time, and hence its peak value increases when $\theta_{0}$ increases. To reduce it to an acceptable value for implementation in missiles with low turning rate, as indicated by Fig. 3, we can decrease the value of parameter to $b=1.0$, at the expense of a longer time for interception. This is achieved in Trajectory 5 in Fig. 2 for the case of $\theta_{0}=$ $\pi / 2$, with a lower initial turning rate than Trajectory 4.

b / In this case, we allow the target to have a constant turning rate by using

$$
\theta_{T}=c \tau \text {. }
$$

Figure 4 shows the missile trajectories with the initial values $\theta_{0}=0$ and $\theta_{0}=\frac{\pi}{2}$

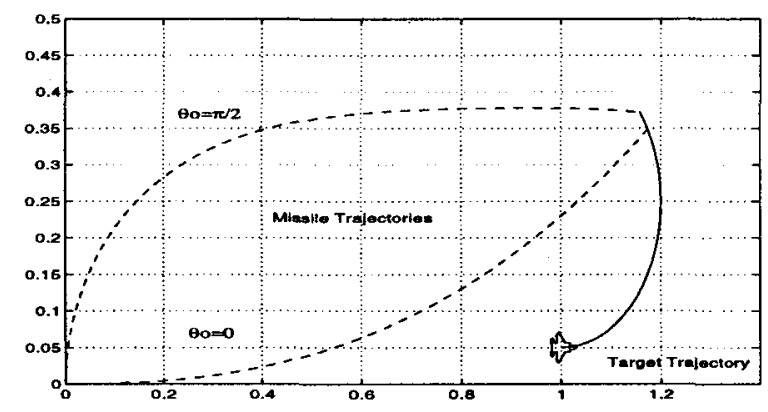

Figure 4: Circular Evasive Trajectory.

For the value of $c=5.0$, both intercept trajectories are achieved with $a=3.0, b=3.0, n=3.0$.

c / In the last example, we consider a sinusoidal change in the heading of the target in the form

$$
\theta_{T}=c \sin \omega \tau \text {. }
$$




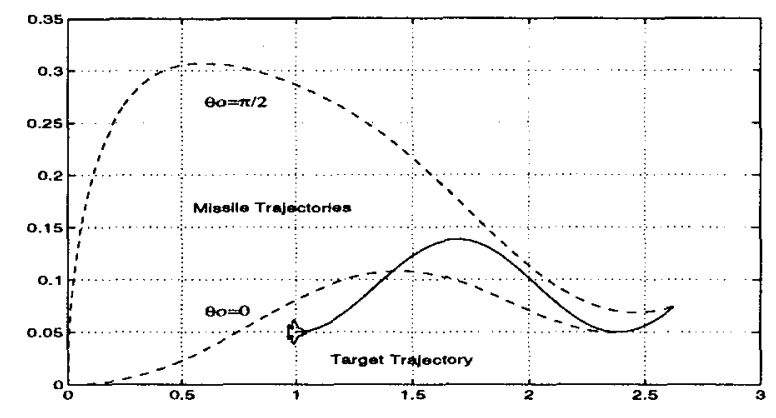

Figure 5: Sinusoidal Evasive Trajectory.

The values used for this evasive trajectory are $c=$ 0.2 and $\omega=4.5$. Both intercept trajectories are achieved with $a=3.0, b=3.0, n=1.701$.

We have experimented with a variety of evasive maneuvers for the target, and it has been found that it is always possible to select the parameters $a$ and $b$ to have successful interception with an acceptable acceleration for the missile. Furthermore, the guidance law tends to tail chase, and hence in the terminal phase, the linearization of equations $(5) \sim(7)$ is valid.

\section{Terminal Guidance}

The control law used in this guidance tends to align the velocity of the missile with the line-of-sight and with the velocity of the target. Hence, in the final phase of the homing, the two angles $(\beta-\theta)$ and $\left(\beta-\theta_{T}\right)$ are reasonably small. This leads to the linearized system for terminal guidance :

$$
\begin{aligned}
& \frac{d r}{d \tau}=(1-n), \\
& \frac{d \beta}{d \tau}=\frac{1}{r}\left[-\left(\beta-\theta_{T}\right)+n(\beta-\theta)\right], \\
& \frac{d \theta}{d \tau}=a \frac{d \beta}{d \tau}+b(\beta-\theta) .
\end{aligned}
$$

The equation for $r$ is decoupled and upon integration, we have the linear relation

$$
r=1-(n-1) \tau \text {. }
$$

For the integration of the other two equations, we change the independent variable from $\tau$ to $r$ to have the new system

$$
\begin{aligned}
& \frac{d \beta}{d r}=-\frac{1}{r} \beta+\frac{n}{(n-1) r} \theta-\frac{1}{(n-1) r} \theta_{T}, \\
& \frac{d \theta}{d r}=-\frac{a(n-1)+b r}{(n-1) r} \beta+\frac{a n+b r}{(n-1) r} \theta-\frac{a}{(n-1) r} \theta_{T} .
\end{aligned}
$$

For the integration of this system, it is convenient to use a new independent variable

$$
z=\frac{b r}{n-1} \text {. }
$$

We notice that since $r$ varies from 1 to zero, this new variable monotonically decreases from $b /(n-1)$ to zero. By changing to $z$, Equations (17) and (18) can be rewritten

$$
\begin{aligned}
& \frac{d \beta}{d z}=-\frac{1}{z} \beta+\frac{n}{(n-1) z} \theta-\frac{1}{(n-1) z} \theta_{T}, \\
& \frac{d \theta}{d z}=-\frac{a+z}{z} \beta+\frac{a n+(n-1) z}{(n-1) z} \theta-\frac{a}{(n-1) z} \theta_{T} .
\end{aligned}
$$

By eliminating $\theta$ between these two equations, we obtain a linear second order differential equation with a forcing function for the variable $\beta$

$z \frac{d^{2} \beta}{d z^{2}}+\left[2-\frac{a n}{n-1}-z\right] \frac{d \beta}{d z}+\frac{1}{(n-1)} \beta=\frac{1}{n-1}\left(\theta_{T}-\frac{d \theta_{T}}{d z}\right)$.

Upon obtaining the solution for $\beta$, the guidance angle $\theta$ for the missile is obtained from

$$
\theta=\frac{(n-1)}{n}\left[\beta+z \frac{d \beta}{d z}+\frac{1}{n-1} \theta_{T}\right] .
$$

The linear equation (22) is a confluent hypergeometric equation ${ }^{[17]}$. Including the forcing function on the right hand side, its general solution is

$$
\begin{aligned}
\beta(z) & =C_{1} M\left(-\frac{1}{n-1}, \frac{2(n-1)-a n}{n-1}, z\right) \\
& +C_{2} U\left(-\frac{1}{n-1}, \frac{2(n-1)-a n}{n-1}, z\right) \\
& +\beta_{p},
\end{aligned}
$$

where $M(\cdot, \cdot, \cdot)$ and $U(\cdot, \cdot, \cdot)$ are linearly independent Kummer's solutions of the homogeneous equation, and $\beta_{p}(z)$ is a particular solution. Using this solution into Eq.(23), we have the solution for $\theta$

$$
\begin{aligned}
\theta(z) & =C_{1} \frac{n-1}{n}\left[M\left(-\frac{1}{n-1}, \frac{2(n-1)-a n}{n-1}, z\right)\right. \\
& \left.-\frac{z}{2(n-1)-a n} M\left(1-\frac{1}{n-1}, 1+\frac{2(n-1)-a n}{n-1}, z\right)\right] \\
& +C_{2} \frac{n-1}{n}\left[U\left(-\frac{1}{n-1}, \frac{2(n-1)-a n}{n-1}, z\right)\right. \\
& \left.+\frac{z}{n-1} U\left(1-\frac{1}{n-1}, 1+\frac{2(n-1)-a n}{n-1}, z\right)\right] \\
& +\frac{n-1}{n}\left[\beta_{p}+z \frac{d}{d z} \beta_{p}+\frac{1}{n-1} \theta_{T}\right] .
\end{aligned}
$$


The constants of integration $C_{1}$ and $C_{2}$ are determined by the initial conditions on $\beta$ and $\theta$.

For a maneuvering target, when the heading $\theta_{T}$ is an arbitrary function of $\tau$, we model it as a polynominal in $\tau$, and ultimately through the change of independent variable by Eqs.(16) and (19), we have $\theta_{T}$ as a polynominal in the independent variable $z$. In this case, the forcing function $f(z)$, which is the right hand side of Eq.(22) is also a polynominal in $z$ and the particular function $\beta_{p}(z)$ in the solutions (24) and (25) for $\beta$ and $\theta$ can be easily obtained by identification.

As an example, we consider the case of a thirddegree polynominal

$$
f(z)=k_{0}+k_{1} z+k_{2} z^{2}+k_{3} z^{3}=\frac{1}{n-1}\left(\theta_{T}-\frac{d \theta_{T}}{d z}\right)
$$

The particular solution of the non-homogeneous equation (22) is assumed to be a polynominal of the same degree

$$
\beta_{p}(z)=\alpha_{0}+\alpha_{1} z+\alpha_{2} z^{2}+\alpha_{3} z^{3}
$$

where the coefficients $\alpha_{i}(i=0,1,2,3)$ are to be found. Upon substituting into Eq.(22) and equating the coefficients of the same power in $z$, we have the solution

$$
\begin{aligned}
\alpha_{3} & =-\frac{k_{3}}{A+3} \\
\alpha_{2} & =\frac{3(B+2) \alpha_{3}-k_{2}}{A+2} \\
\alpha_{1} & =\frac{2(B+1) \alpha_{2}-k_{1}}{A+1} \\
\alpha_{0} & =\frac{B \alpha_{1}-k_{0}}{A}
\end{aligned}
$$

where

$$
\begin{aligned}
A & =-\frac{1}{n-1} \\
B & =\frac{2(n-1)-a n}{n-1}
\end{aligned}
$$

It is not difficult to show that if the forcing function is a polynominal of degree $m$, then in the solution for $\beta_{p}(z)$

$$
\beta_{p}(z)=\alpha_{0}+\alpha_{1} z+\alpha_{2} z^{2}+\alpha_{3} z^{3}+\ldots+\alpha_{m} z^{m}
$$

we first calculate the coefficient $\alpha_{m}$

$$
\alpha_{m}=-\frac{k_{m}}{A+m}
$$

and for any other coefficient $\alpha_{q}(q=0, \ldots, m-1)$, we use the recursive formula

$$
\alpha_{q}=\frac{(q+1)(B+q) \alpha_{q+1}-k_{q}}{A+q} .
$$

As an example, we consider the case of a constant turning rate for the target as given in Eq.(11). Then, in terms of $z$, we have

$$
\theta_{T}=c\left(\frac{1}{n-1}-\frac{z}{b}\right)
$$

The solution $\beta_{p}(z)$ is also a linear function and the coefficients $\alpha_{1}$ and $\alpha_{0}$ are easily calculated from Eqs.(31) and (32). With $n=1.701$, initial values $\theta_{0}=0$ and $\beta_{0}=1 / 20$ and using again $a=3, b=3$, we generate two evasive trajectories for $c=0.06$ and $c=-0.06$. We calculate the intercept trajectories, first by integrating the non-linear equations, and then by using the analytical solution of the linearized system. The two solutions are in excellent agreement with each other, as seen in Fig.6.

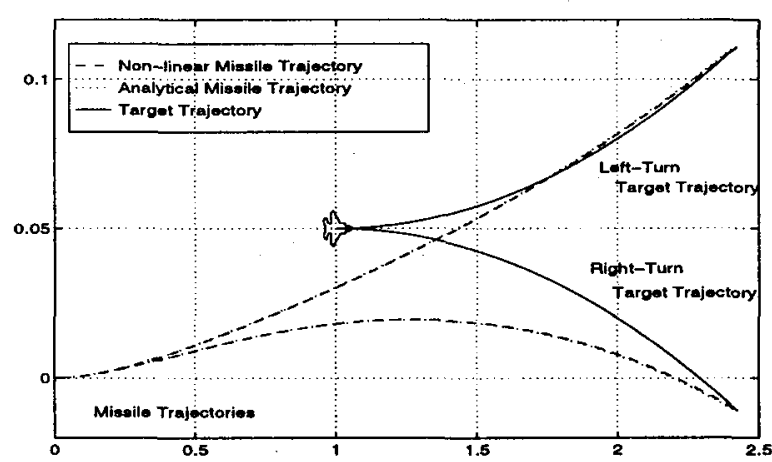

Figure 6: Circular Evasive Trajectory.

In the next example, we model the heading change of a sinusoidal evasive trajectory as a polynominal in $\tau$.

$$
\theta_{T}(\tau)=0.38 \tau^{3}-0.827 \tau^{2}+0.4 \tau
$$

After changing the independent variable, again, using the value $n=1.701, a=3, b=3$ with the trinominal in $z$

$$
\theta_{T}(z)=-0.01407 z^{3}-0.08880 z^{2}-0.12010 z-0.00928,
$$


for the heading of the target, the analytical solution obtained is in perfect agreement with the numerical solution of the non-linear system. This is shown in Fig.7.

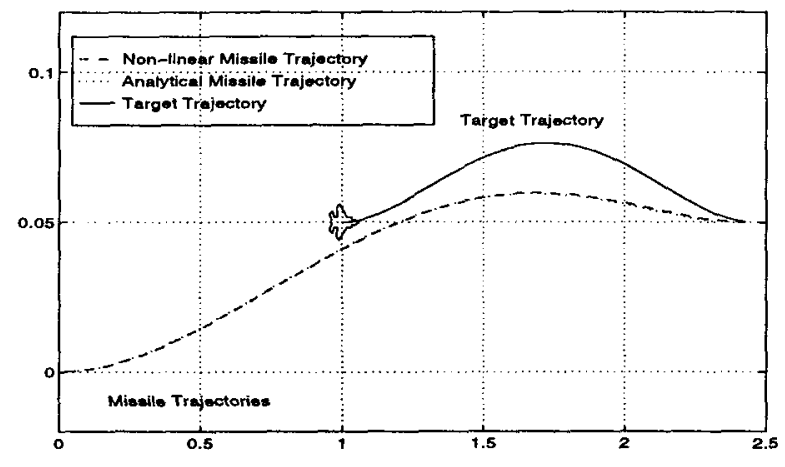

Figure 7: Sinusoidal Evasive Trajectory.

\section{Conclusion}

In this paper, a new guidance law, which combines pursuit guidance and proportional navigation has been proposed. This guidance law depends on two parameters that determine the relative importance of pursuit guidance and proportional navigation. Numerical simulations of the nonlinear equations of motion have been presented and suggest that the parameters of this law can be chosen to reduce the peak value of the missile acceleration. An analysis of the terminal portion of the trajectory has been presented, leading to a confluent hypergeometric equation. A closed form solution of this equation has been obtained in the general case where the target performs maneuvers such that its heading angle is a polynomial function of time. The analytic solution based on linearization and the numerical simulation of the nonlinear equations show good agreement.

\section{References}

[1] Locke, A. S., Guidance, D.Van Nostrand Co., Princeton, 1955, Chap.12.

[2] Howe, R. M., "Guidance," System Engineering Handbook, edited by R. E. Machol, W. P. Tanner Jr., and S. N. Alexander, McGraw-Hill, 1965, Chap.19.

[3] Pastrick, H. L., Seltzer, S. M., and Warren, M. E., Guidance Laws for Short-Range Tactical Missiles, Journal of Guidance and Control, Vol.4, No.2, March-April 1981, pp.98-108.
[4] Gonzalez, J., New Methods in the Terminal Guidance and Control of Tactical Missiles, NATO AGARD Lecture Series, Vol.101, 1979, pp.3-1 3-23.

[5] Kabamba, P. T., Guidance and Navigation Lecture Notes, The University of Michigan, 1995.

[6] Vinh, N. X., Integral Performance of Supersonic Aircraft. Technical Report(4), The University of Michigan, 1994.

[7] Zarchan, P., Tactical and Strategic Missile Guidance Second Edition, AIAA, 1994.

[8] Lin, Ching-Fang, Modern Navigation, Guidance, and Control Processing, Prentice-Hall, 1991, Sec. 6.5.

[9] Guelman, M., The Closed-Form Solution of True Proportional Navigation, IEEE Transactions on Aerospace and Electronic Systems, Vol.12, No.4, July 1976, pp.472-482.

[10] Shukla, U. S., and Mahapatra, P. R., Generalized Linear Solution of Proportional Navigation, IEEE Transactions on Aerospace and Electronic Systems, Vol.24, No.3, May 1988, pp.231-238.

[11] Mahapatra, P. R. and Shukla, U. S., Accurate Solution of Proportional Navigation for Maneuvering Targets, IEEE Transactions on Aerospace and Electronic Systems, Vol.25, No.1, January 1989, pp.81-89.

[12] Becker, K., Closed-Form Solution of Pure Proportional Navigation, IEEE Transactions on Aerospace and Electronic Systems, Vol.26, No.3, May 1990, pp.526-533.

[13] Cochran Jr., J. E., No, T. S., and Thaxton, D. G., Analytical Solutions to a Guidance Problem, Journal of Guidance, Control and Dynamics, Vol.14, No.1, Jan.-Feb. 1991, pp.117-122.

[14] Yuan., P. J. and Chern, J. S., Solutions of True Proportional Navigation for Maneuvering and Nonmaneuvering Targets, Journal of Guidance, Control and Dynamics, Vol.15, No.1, Jan.- Feb. 1992, pp.268-271.

[15] Yuan., P. J. and Hsu, S. C., Exact Closed-Form Solution of Generalized Proportional Navigation, Journal of Guidance, Control and Dynamics, Vol.16, No.5, Sep.- Oct. 1993, pp.963-966. 
[16] Balakrishnan, S. N., Analytical Missile Guidance Law with a Time-Varying Transformation, Journal of Guidance, Control and Dynamics, Vol.19, No.2, Mar.-Apr. 1996, pp.496-499.

[17] Abramowitz, M. and Stegun, I. A., Handbook of Mathematical Functions, National Bureau of Standard, 1964. 